BMJ Open

Diabetes

Research

\& Care

\title{
Conditions, pathogenesis, and progression of diabetic kidney disease and early decliner in Japan
}

Yui Yoshida (D) , ${ }^{1,2}$ Kosuke Kashiwabara, ${ }^{1,3}$ Yosuke Hirakawa, ${ }^{2}$ Tetsuhiro Tanaka, ${ }^{2}$ Shinsuke Noso (1) , ${ }^{4}$ Hiroshi Ikegami (D) , ${ }^{4}$ Mitsuru Ohsugi, ${ }^{5}$ Kohjiro Ueki, ${ }^{6}$ Tomoya Mita (D) , ${ }^{7}$ Hirotaka Watada, ${ }^{7}$ Daisuke Koya, ${ }^{8}$ Koki Mise, ${ }^{9}$ Jun Wada (D) , ${ }^{9}$ Miho Shimizu, ${ }^{10}$ Takashi Wada, ${ }^{11}$ Yumi Ito, ${ }^{12}$ Ichiei Narita, ${ }^{12}$ Naoki Kashihara, ${ }^{13}$ Masaomi Nangaku, ${ }^{2}$ Yutaka Matsuyama ${ }^{1}$

To cite: Yoshida Y, Kashiwabara K, Hirakawa Y, et al. Conditions, pathogenesis, and progression of diabetic kidney disease and early decliner in Japan. BMJ Open Diab Res Care 2020;8:e000902. doi:10.1136/ bmjdrc-2019-000902

- Additional material is published online only. To view please visit the journal online (http://dx.doi.org/10.1136/ bmjdrc-2019-000902).

Received 16 0ctober 2019 Revised 15 January 2020 Accepted 14 February 2020
Check for updates

\section{(C) Author(s) (or their} employer(s)) 2020. Re-use permitted under CC BY-NC. No commercial re-use. See rights and permissions. Published by BMJ.

For numbered affiliations see end of article.

Correspondence to Dr Masaomi Nangaku; mnangaku-tky@umin.ac.jp

\section{ABSTRACT}

Objective Glomerular filtration rate (GFR) decreases without or prior to the development of albuminuria in many patients with diabetes. Therefore, albuminuria and/or a low GFR in patients with diabetes is referred to as diabetic kidney disease (DKD). A certain proportion of patients with diabetes show a rapid progressive decline in renal function in a unidirectional manner and are termed early decliners. This study aimed to elucidate the prevalence of DKD and early decliners and clarify their risk factors.

Research design and methods This combination cross-sectional and cohort study included 2385 patients with diabetes from 15 hospitals. We defined DKD as a urinary albumin to creatinine ratio (ACR) $\geq 30 \mathrm{mg} / \mathrm{gCr}$ and/or estimated glomerular filtration rate (eGFR) $<60$ $\mathrm{mL} / \mathrm{min} / 1.73 \mathrm{~m}^{2}$. We classified patients into four groups based on the presence or absence of albuminuria and a decrease in eGFR to reveal the risk factors for DKD. We also performed a trajectory analysis and specified the prevalence and risk factors of early decliners with sequential eGFR data of 1955 patients in five facilities. Results of our cohort, $52 \%$ had DKD. Above all, $12 \%$ with a low eGFR but no albuminuria had no traditional risk factors, such as elevated glycated hemoglobin, elevated blood pressure, or diabetic retinopathy in contrast to patients with albuminuria but normal eGFR. Additionally, $14 \%$ of our patients were early decliners. Older age, higher basal eGFR, higher ACR, and higher systolic blood pressure were significantly associated with early decliners.

Conclusions The prevalence of DKD in this cohort was larger than ever reported. By testing eGFR yearly and identifying risk factors in the early phase of diabetes, we can identify patients at high risk of developing end-stage renal disease.

\section{INTRODUCTION}

The prevalence of diabetes mellitus has increased worldwide, and 425 million adult patients had diabetes in $2017 .{ }^{1}$ Similarly, the prevalence of diabetes mellitus in Japan has increased, and the number of the patients was the largest ever, approximately 10 million in 2016. ${ }^{2}$ The prevalence of diabetic nephropathy has increased accordingly, and it has

\section{Significance of this study}

What is already known about this subject?

- The concepts of "diabetic kidney disease (DKD)" and "early decliners" among patients with diabetes have become well known, but the actual conditions and risk factors of them remain ambiguous.

What are the new findings?

- We revealed the prevalence and risk factors of DKD with 2385 patients with diabetes in Japan and identified the pattern of estimated glomerular filtration rate (eGFR) variation and risk factors of early decliners during approximately 3 years of follow-up.

How might these results change the focus of research or clinical practice?

- By testing eGFR yearly and identifying risk factors in the early phase of diabetes, we can identify patients at high risk of developing end-stage renal disease.

remained the main cause of incident dialysis since 1998 in Japan. ${ }^{3}$ However, details of the cause and progress of diabetic nephropathy are unclear. We must grasp the actual condition of patients with diabetes and formulate intervention methods to prevent renal damage from progressing to end-stage renal disease (ESRD).

This study examined diabetic kidney disease (DKD), which is a clinical diagnosis of diabetic damage due to diabetes mellitus. Classical course of formerly known diabetic nephropathy is now changed, possibly due to widespread use of renin-angiotensin-aldosterone system (RAAS) inhibitors and presence of multiple comorbid conditions. The typical clinical course of classical diabetic nephropathy is as follows: microalbuminuria develops, progresses to macroalbuminuria and sometimes leads to nephrotic syndrome. Eventually, the glomerular filtration rate (GFR) 
decreases and patients develop ESRD. However, recent epidemiological studies showed that many patients with diabetes develop decreased GFR without albuminuria. ${ }^{4-6}$

In the light of the fact that a certain proportion of patients with diabetes do not show the typical clinical course, those whose albuminuria is $\geq 30 \mathrm{mg} / \mathrm{gCr}$ and/ or estimated GFR (eGFR) is $<60 \mathrm{~mL} / \mathrm{min} / 1.73 \mathrm{~m}^{2}$ and whose primary disease is diabetes are now considered to have DKD. ${ }^{7}$

Furthermore, a certain number of patients with diabetes show rapid declines in kidney function in a unidirectional process and are termed early decliners. ${ }^{8-10}$ Previous studies defined early decliners as patients with type 2 diabetes with normal baseline eGFR $(\geq 60 \mathrm{~mL} /$ $\left.\mathrm{min} / 1.73 \mathrm{~m}^{2}\right) .{ }^{10} 11$ They suggested that the eGFR slope of early-stage patients with type 2 diabetes could predict the future risk of ESRD. ${ }^{11}$

Here we aimed to solve two problems that hinder us from figuring out the actual condition of DKD. One problem is that the prevalence and characteristics of albuminuric versus non-albuminuric patients with DKD are unknown, while the other is that the definition and risk factors of early decliners remain ambiguous. Because the population of diabetes and number of deaths due to diabetes are the highest in Western Pacific region including Japan in the world, our research has important meaning as a representative of the region. ${ }^{1}$

For this reason, we integrated multicenter cohort data to determine the definition, prevalence, and risk of DKD onset, progression, and early decline in patients with diabetes in Japan.

\section{MATERIALS AND METHODS}

\section{Patients}

The data of a total of 9342 patients with diabetes attending 15 hospitals were collected and incorporated in each cohort (online supplementary appendix). Patients were diagnosed with diabetes by Japanese guidelines ${ }^{12}$ with each facility. A cross-sectional study of patients attending all 15 hospitals for the analysis of DKD prevalence was performed first (figure 1).

Next, a longitudinal analysis using sequential eGFR data of patients from 12 facilities was performed to identify early decliners. After excluding patients who started maintenance dialysis during the follow-up period, the analysis was started at a point at which the patients' urinary albumin to creatinine ratio (ACR)/urinary protein creatinine ratio (PCR) and eGFR data existed. Patient data within 4 years from baseline were included for trajectory analysis.

\section{Clinical and laboratory data}

The participants' clinical parameters including age, sex, height, weight, systolic blood pressure (SBP), and diastolic blood pressure were recorded at least once each year in most hospitals. Their clinical examination items were determined independently at each hospital. Four hospitals had medication data (online supplementary appendix). Body mass index (BMI) was calculated by dividing the weight (kilogram) by height squared $\left(\mathrm{m}^{2}\right)$. The eGFR was calculated using the Modification of Diet in Renal Disease formula adjusted for Japanese ethnicity: eGFR $\left(\mathrm{mL} / \mathrm{min} / 1.73 \mathrm{~m}^{2}\right)=194 \times \mathrm{Cr}^{-1.094} \times$ age $^{-0.287}$ for males and eGFR $\left(\mathrm{mL} / \mathrm{min} / 1.73 \quad \mathrm{~m}^{2}\right)=194 \times \mathrm{Cr}^{-}$ ${ }^{1.094} \times$ age $^{-0.287} \times 0.739$ for females. ${ }^{13}$ If low-density lipoprotein (LDL) cholesterol was not measured, we calculated it as follows: LDL cholesterol=total cholesterol-highdensity lipoprotein (HDL) cholesterol-triglycerides (TG)*0.2. This formula was applied only in the range of TG level $<400 \mathrm{mg} / \mathrm{dL}$. Because there was a high correlation between LDL cholesterol and total cholesterol, and because LDL cholesterol was available in fewer patients

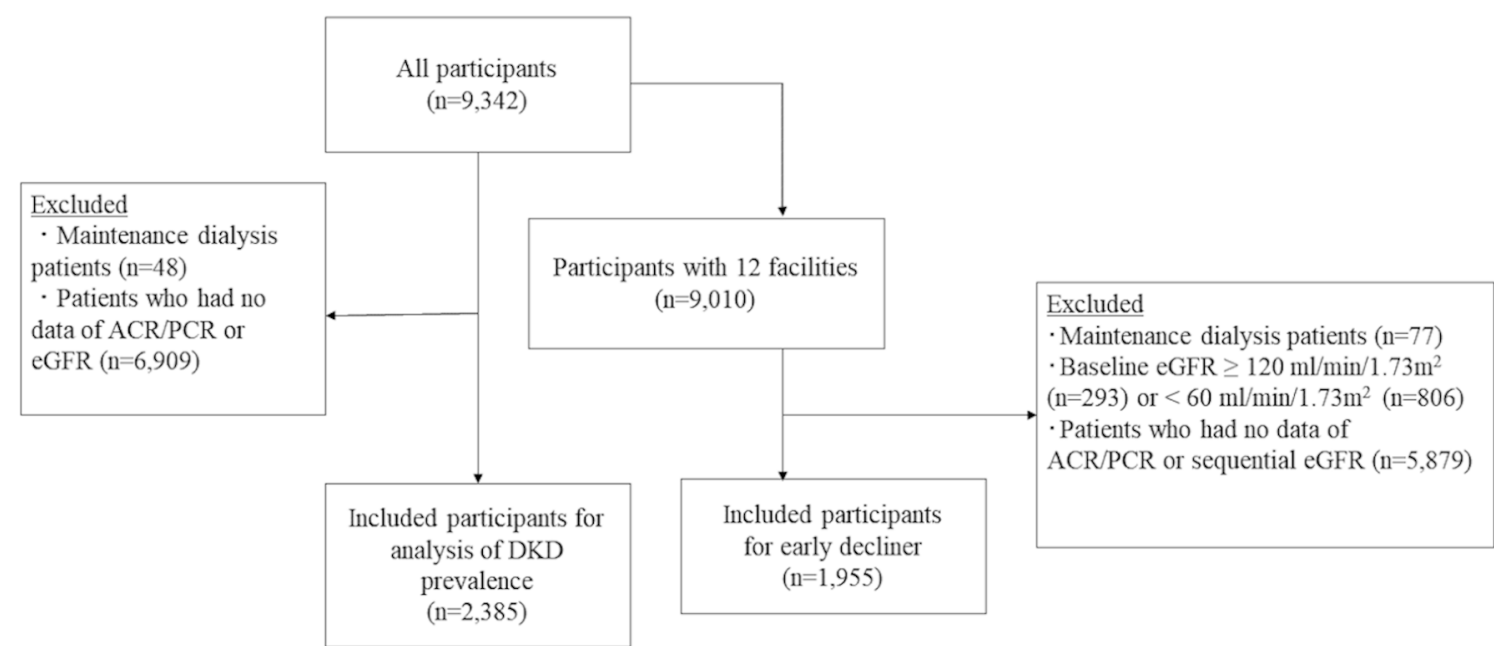

Figure 1 Participant selection for each analysis. The patients' data from all facilities were used in the cross-sectional analysis to reveal the prevalence and risk factors of DKD. Then, to examine the characteristics of early decliners, the data of patients who had normal baseline and sequential eGFR levels were subjected to the longitudinal analysis. ACR, urinary albumin to creatinine ratio; DKD, diabetic kidney disease; eGFR, estimated glomerular filtration rate; PCR, urinary protein to creatinine ratio. 
Table 1 Baseline variables sorted by albuminuria and eGFR $(n=2385)$

\begin{tabular}{|c|c|c|c|c|c|}
\hline & $\begin{array}{l}\text { No DKD } \\
(n=1155)\end{array}$ & $\begin{array}{l}\text { Low eGFR† group } \\
(n=281)\end{array}$ & $\begin{array}{l}\text { Albuminuria } \neq \text { group } \\
(n=514)\end{array}$ & $\begin{array}{l}\text { Low eGFR and } \\
\text { albuminuria group } \\
(n=435)\end{array}$ & $\begin{array}{l}\text { Overall } \\
(\mathrm{N}=2385)\end{array}$ \\
\hline Male & $714(62 \%)$ & $171(61 \%)$ & $333(65 \%)$ & $280(64 \%)$ & $1498(63 \%)$ \\
\hline Age (years) & $62(52-70)$ & $70(65-76)^{\star \star \star}$ & $61(54-69)$ & $66(59-75)^{\star \star \star}$ & $64(55-71)$ \\
\hline BMI (kg/m²) & $24(22-27)$ & $25(23-28)^{*}$ & $25(22-28)^{\star \star}$ & $25(23-28)^{\star *}$ & $24(22-27)$ \\
\hline Serum creatinine $(\mathrm{mg} / \mathrm{dL})$ & $0.69(0.6-0.81)$ & $1.02(0.88-1.14)^{\star \star \star}$ & $0.71(0.6-0.83)^{\star}$ & $1.2(1.02-1.6)^{\star \star \star}$ & $0.79(0.64-0.96)$ \\
\hline Total cholesterol (mg/dL) & $184(163-207)$ & $177(156-197)^{\star *}$ & $189(166-211)$ & $187(162-217)$ & 185 (162-209) \\
\hline LDL cholesterol (mg/dL) & $104(86-123)$ & $100(79-116)^{\star \star}$ & $104(86-124)$ & $97(81-114)^{\star \star \star}$ & $102(84-121)$ \\
\hline HDL cholesterol (mg/dL) & $52(43-64)$ & $51(42-62)$ & $51(43-61)$ & $49(42-62)$ & $51(43-63)$ \\
\hline Triglycerides (mg/dL) & $110(77-164)$ & $115(88-163)$ & $126(86-182)^{\star \star \star}$ & $121(92-181)^{\star \star \star}$ & $116(83-170)$ \\
\hline $\mathrm{HbA} 1 \mathrm{c} \S(\%)$ & $7.1(6.6-7.9)$ & $6.9(6.5-7.5)^{\star *}$ & $7.6(6.8-8.7)^{\star \star \star}$ & $6.9(6.3-7.7)^{\star \star \star}$ & $7.1(6.5-8.0)$ \\
\hline $\begin{array}{l}\text { Diastolic blood pressure } \\
(\mathrm{mm} \mathrm{Hg})\end{array}$ & $73(66-80)$ & $70(64-76)^{\star \star}$ & $77(69-84)^{\star \star \star}$ & $73(65-80)$ & $73(66-81)$ \\
\hline $\begin{array}{l}\text { History of diabetic } \\
\text { retinopathy (yes/no) }\end{array}$ & $142(22 \%)$ & $44(27 \%)$ & $145(53 \%)^{\star \star \star}$ & $216(66 \%)^{\star \star \star}$ & $547(22.9 \%)$ \\
\hline
\end{tabular}

The values are expressed in $n(\%)$ or median (IQR).

${ }^{*} \mathrm{p}<0.05,{ }^{* *} \mathrm{p}<0.01,{ }^{* * *} \mathrm{p}<0.001$.

teGFR $<60 \mathrm{~mL} / \mathrm{min} / 1.73 \mathrm{~m}^{2}$ and normoalbuminuria (ACR $<30 \mathrm{mg} / \mathrm{gCr}$ ).

$\ddagger A C R \geq 30 \mathrm{mg} / \mathrm{gCr}$ and normal eGFR $\left(\geq 60 \mathrm{~mL} / \mathrm{min} / 1.73 \mathrm{~m}^{2}\right)$.

$\S N G S P$, National Glycohemoglobin Standardization Program.

ACR, urinary albumin to creatinine ratio; BMI, body mass index; DKD, diabetic kidney disease; eGFR, estimated glomerular filtration rate; HbA1c, glycated hemoglobin; HDL, high-density lipoprotein; LDL, low-density lipoprotein.

than total cholesterol, we set the main results as using total cholesterol instead of LDL cholesterol, and added the results with LDL cholesterol instead of total cholesterol in online supplementary tables. PCR was calculated by taking the ratio between urinary protein and urinary creatinine in occasional urine sample. In this study, PCR $\geq 150 \mathrm{mg} / \mathrm{gCr}$ and PCR $\geq 500 \mathrm{mg} / \mathrm{gCr}$ are considered equal to ACR $\geq 30 \mathrm{mg} / \mathrm{gCr}$ and $\mathrm{ACR} \geq 300 \mathrm{mg} / \mathrm{gCr}$, respectively. ${ }^{13}$

\section{Statistical analysis}

Variables are expressed as percentages for categorical data and as median and IQR (25th-75th percentiles) for continuous data. Continuous variables were compared with no DKD category by Mann-Whitney U test, and binary variables were compared with no DKD category by $\chi^{2}$ test (table 1 ).

For analytical purposes with cross-sectional analysis for DKD, patients were divided into four groups according to their ACR $(<30 \mathrm{mg} / \mathrm{gCr}$ or $\geq 30 \mathrm{mg} / \mathrm{gCr})$ and eGFR $\left(<60 \mathrm{~mL} / \mathrm{min} / 1.73 \mathrm{~m}^{2}\right.$ or $\left.\geq 60 \mathrm{~mL} / \mathrm{min} / 1.73 \mathrm{~m}^{2}\right)$, the threshold level of microalbuminuria, and decreased eGFR, respectively. ${ }^{14}$ Risk factors associated with each DKD category were calculated by logistic regression analysis in contradistinction to no patients with DKD.

Next, using sequential eGFR data of the 12 facilities, longitudinal analyses such as multiple linear regression analysis, trajectory analysis, and polytomous logistic regression analysis were performed. To determine yearly eGFR decline rate, we calculated the differences between baseline and final measurements and divided them by the follow-up period. Patients whose measurement intervals were within 3 months were excluded.

In addition, a multiple linear regression analysis was performed of the yearly change of eGFR to identify the baseline risk factors weighting by the square of the follow-up period.

In the trajectory analysis, the patients were classified into three groups; the group whose eGFR decreased most was defined as the early decliner group. A consequential variable of patients was sorted to plural subgroups to which every patient potentially belonged by trajectory analysis. ${ }^{15}$ Additionally, their risk factors were calculated by polytomous logistic regression analysis.

Statistical analyses were performed using SAS (V.9.4; SAS Institute, Cary, North Carolina, USA). Values of $\mathrm{p}<0.05$ were defined as statistically significant.

We shows the details of the Ethics Committee Approval about this study in the online supplementary appendix.

\section{RESULTS}

\section{Baseline characteristics and prevalence of DKD}

After the exclusion of patients on maintenance dialysis or whose ACR/PCR or eGFR data were missing at the time of registration, 2385 patients were included in 
a cross-sectional study (figure 1). The overall median patient age was 64 (IQR, 55-71) years (table 1); $62.8 \%$ of them were males. The median serum creatinine level was $0.79 \mathrm{mg} / \mathrm{dL}$ (IQR, 0.64-0.96 mg/dL). Type 1 diabetes accounted for $7.8 \%$, while type 2 diabetes accounted for $91 \%$; other conditions such as liver or pancreas disorders, drugs or chemical substances, and genetic abnormalities accounted for $1.3 \%$. The baseline variables with each type of diabetes are shown in online supplementary tables S1 and S2.

All participants were divided into four groups according to their ACR $(<30 \mathrm{mg} / \mathrm{g}$ or $\geq 30 \mathrm{mg} / \mathrm{g})$ and eGFR $(<60$ $\mathrm{mL} / \mathrm{min} / 1.73 \mathrm{~m}^{2}$ or $\left.\geq 60 \mathrm{~mL} / \mathrm{min} / 1.73 \mathrm{~m}^{2}\right)$. The prevalence of DKD was $52 \%$ in this study. Albuminuria was documented in $40 \%$ of the patients, and $30 \%$ of the patients had a decreased eGFR $\left(<60 \mathrm{~mL} / \mathrm{min} / 1.73 \mathrm{~m}^{2}\right)$ (online supplementary figure 1 ). While $28 \%$ of patients with type 1 diabetes had $\mathrm{DKD}, 54 \%$ of patients with type 2 diabetes had DKD (online supplementary table S3).

\section{Risk factors associated with albuminuria/decreased eGFR}

To reveal the risk factors associated with the three DKD categories, we performed logistic regression analyses compared with patients with no DKD. In this analysis, patients with diabetes other than type 1 or type 2 were excluded. Risk factors for a decreased eGFR $(<60 \mathrm{~mL} /$ $\min / 1.73 \mathrm{~m}^{2}$ ) group, albuminuria group, and both decreased eGFR and albuminuria group were as follows: higher uric acid (UA) level, older age, and lower total cholesterol level for the decreased eGFR group; a history of diabetic retinopathy was associated with the highest OR, followed by a higher level of glycated hemoglobin (HbAlc) and SBP for the albuminuria group; female sex, older age, higher UA and SBP levels, and history of diabetic retinopathy for the decreased eGFR and albuminuria group (table 2). After excluding the patients from the two hospitals that performed the renal biopsy for all participants, the same trend was indicated (online supplementary table S4). Although we did the same analysis using LDL cholesterol as a variable instead of total cholesterol (online supplementary table S5), originally significant variables still remained significant.

\section{Prescribed medicines}

Online supplementary table S6 shows the prescription drugs' information of the four hospitals. Compared with patients with no DKD, patients in the low eGFR or albuminuria group were prescribed antihypertensive drugs more frequently. On the other hand, patients of the albuminuria and normal eGFR category were more often treated with hypoglycemic drugs and insulin, antidyslipidemic drugs, and antihypertensive drugs than patients with no DKD.

\section{Definition and risk factors of early decliner}

To determine the characteristics of early decliners, a longitudinal analysis was performed. Patients who started maintenance dialysis during the follow-up period were excluded because their eGFR could not reflect their renal function after the start of maintenance dialysis. We started the analysis at the point at which the patients' oldest data of ACR/PCR and eGFR existed. A total of 2761 patients from 12 facilities had sequential eGFR data and at least a single ACR/PCR measurement and did not experience a temporal increase in eGFR due to

Table 2 Risk factors of DKD by category

\begin{tabular}{|c|c|c|c|c|c|c|}
\hline \multirow[b]{2}{*}{ Variables } & \multicolumn{2}{|c|}{ Low eGFR group* } & \multicolumn{2}{|c|}{ Albuminuria group $\dagger$} & \multicolumn{2}{|c|}{$\begin{array}{l}\text { Low eGFR and albuminuria } \\
\text { group }\end{array}$} \\
\hline & OR & $95 \% \mathrm{Cl}$ & OR & $95 \% \mathrm{Cl}$ & OR & $95 \% \mathrm{Cl}$ \\
\hline Sex (female) & 1.37 & 0.81 to 2.32 & 0.99 & 0.64 to 1.51 & 1.81 & 1.11 to 2.95 \\
\hline Age (by 10 years) & 2.69 & 2.03 to 3.58 & 1.18 & 1.00 to 1.40 & 1.77 & 1.41 to 2.21 \\
\hline BMI (by $5 \mathrm{~kg} / \mathrm{m}^{2}$ ) & 1.31 & 0.95 to 1.81 & 1.22 & 0.97 to 1.53 & 1.25 & 0.94 to 1.66 \\
\hline Total cholesterol (by $10 \mathrm{mg} / \mathrm{dL}$ ) & 0.91 & 0.83 to 0.997 & 0.93 & 0.87 to 1.00 & 1.02 & 0.95 to 1.10 \\
\hline HDL cholesterol (by $10 \mathrm{mg} / \mathrm{dL}$ ) & 0.99 & 0.81 to 1.21 & 1.02 & 0.88 to 1.18 & 0.95 & 0.79 to 1.13 \\
\hline Triglycerides (by $10 \mathrm{mg} / \mathrm{dL}$ ) & 1 & 0.97 to 1.04 & 1.01 & 0.99 to 1.04 & 1.01 & 0.98 to 1.04 \\
\hline HbA1c $(\%)$ & 1.05 & 0.81 to 1.36 & 1.29 & 1.11 to 1.51 & 0.78 & 0.60 to 1.004 \\
\hline Uric acid (mg/dL) & 1.83 & 1.48 to 2.27 & 1.13 & 0.96 to 1.32 & 2.04 & 1.68 to 2.48 \\
\hline SBP (by 10 mm Hg) & - & - & 1.32 & 1.17 to 1.48 & 1.2 & 1.05 to 1.38 \\
\hline DBP (by 10 mm Hg) & 1.06 & 0.83 to 1.34 & - & - & - & - \\
\hline Diabetic retinopathy (yes/no) & 1.23 & 0.72 to 2.10 & 2.17 & 1.46 to 3.21 & 3.83 & 2.44 to 6.03 \\
\hline
\end{tabular}

Logistic regression analysis with each DKD group in contradistinction to no DKD group to find related risk factors.

*eGFR $<60 \mathrm{~mL} / \mathrm{min} / 1.73 \mathrm{~m}^{2}$ and normoalbuminuria (ACR $<30 \mathrm{mg} / \mathrm{gCr}$ ).

†ACR $\geq 30 \mathrm{mg} / \mathrm{gCr}$ and normal eGFR $\left(\geq 60 \mathrm{~mL} / \mathrm{min} / 1.73 \mathrm{~m}^{2}\right)$.

$\ddagger$ NGSP, National Glycohemoglobin Standardization Program.

ACR, urinary albumin to creatinine ratio; BMI, body mass index; DBP, diastolic blood pressure; DKD, diabetic kidney disease; eGFR, estimated glomerular filtration rate; HbA1c, glycated hemoglobin; HDL, high-density lipoprotein; SBP, systolic blood pressure. 
Table 3 Baseline risk factors of eGFR decline on multiple regression analysis and the rapidly lowering group (group 1) by trajectory analysis

\begin{tabular}{|c|c|c|c|c|}
\hline \multirow[b]{2}{*}{ Variables } & \multicolumn{2}{|c|}{ Multiple regression analysis } & \multicolumn{2}{|c|}{ Trajectory analysis } \\
\hline & Regression coefficient & $95 \% \mathrm{Cl}$ & OR & $95 \% \mathrm{Cl}$ \\
\hline Sex (female) & 0.036 & -0.44 to 0.52 & 1.09 & 0.78 to 1.52 \\
\hline Age (by 10 years) & -0.44 & -0.65 , to -0.23 & 1.38 & 1.19 to 1.60 \\
\hline BMI (by $5 \mathrm{~kg} / \mathrm{m}^{2}$ ) & -0.0098 & -0.27 to 0.25 & 0.98 & 0.87 to 1.09 \\
\hline HDL cholesterol (by 10 mg/dL) & 0.11 & -0.057 to 0.28 & 0.95 & 0.84 to 1.07 \\
\hline Total cholesterol (by $10 \mathrm{mg} / \mathrm{dL}$ ) & -0.034 & -0.11 to 0.046 & 1.02 & 0.96 to 1.07 \\
\hline Triglycerides (by 10 mg/dL) & -0.021 & -0.052 to 0.010 & 1.02 & 0.998 to 1.03 \\
\hline $\mathrm{HbA} 1 \mathrm{c}^{*}(\%)$ & 0.031 & -0.18 to 0.24 & 1.10 & 0.97 to 1.26 \\
\hline Uric acid (mg/dL) & 0.050 & -0.16 to 0.26 & 1.03 & 0.89 to 1.20 \\
\hline Systolic blood pressure (by $10 \mathrm{~mm} \mathrm{Hg}$ ) & -0.17 & -0.30 to -0.038 & 1.05 & 0.95 to 1.15 \\
\hline ACR (by 10 mg/gCr) & -0.019 & -0.027 to -0.010 & 1.004 & 1.001 to 1.008 \\
\hline eGFR (by $10 \mathrm{~mL} / \mathrm{min} / 1.73 \mathrm{~m}^{2}$ ) & -0.85 & -1.03 to -0.68 & 1.86 & 1.65 to 2.11 \\
\hline
\end{tabular}

Calculated by multiple regression analysis as to yearly change of eGFR to identify the baseline risk factors weighting by the square of followup period.

Calculated by multiple logistic regression analysis in contradistinction to moderate lowering group (group 2).

*NGSP, National Glycohemoglobin Standardization Program.

ACR, urinary albumin to creatinine ratio; BMI, body mass index; eGFR, estimated glomerular filtration rate; HbA1c, glycated hemoglobin;

HDL, high-density lipoprotein.

hyperfiltration (figure 1). Their baseline variables are shown in online supplementary table S7.

The baseline characteristics of participants in the analysis of early decliners was almost equal to those of participants in the cross-sectional analysis. The mean eGFR follow-up period was 3.0 years. Online supplementary table $\mathrm{S} 8$ shows the eGFR decline rate sorted by diabetes type and baseline ACR and eGFR. Especially in patients with type 2 diabetes, the eGFR decline rate of the decreased eGFR and normoalbuminuric group was significantly slower than that of patients with no DKD. On the other hand, the eGFR decline rate of the albuminuria and normal eGFR group was significantly higher than that of the no DKD group.
Risk factors of early decliners with a normal baseline eGFR $\left(\geq 60 \mathrm{~mL} / \mathrm{min} / 1.73 \mathrm{~m}^{2}\right)$ were calculated by multiple regression analysis with eGFR variation (table 3 ). Older age and higher baseline levels of SBP, eGFR, and ACR were significantly associated.

Moreover, normal eGFR patients were divided into three groups to define early decliners by trajectory analysis (figure 2), and we identified that patterns of eGFR variation were classified as a rapid lowering group (group 1), moderate lowering group (group 2), and gradually rising group (group 3). All posterior probabilities with each group were as high as $85 \%$ or more (online supplementary table S10). The trajectories of groups 1 and 3 were approximated by quadratic curve, while that of group 2 was

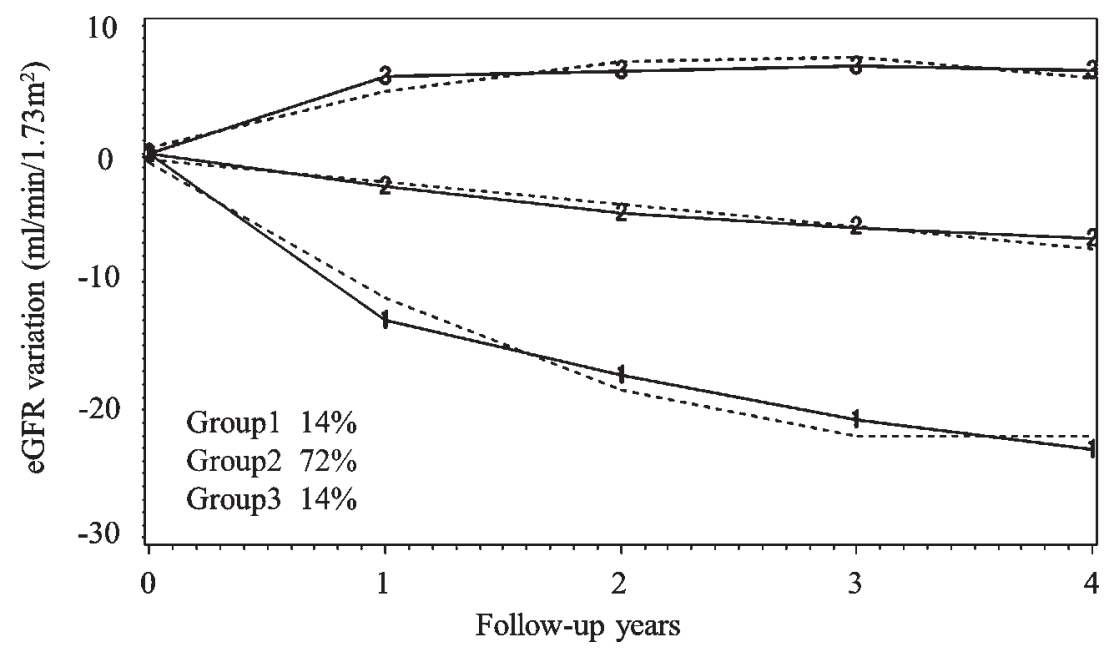

Figure 2 Yearly estimated glomerularfiltration rate (eGFR) variation divided into three groups and modeled in a trajectory analysis. The solid lines show the actual transit, while the broken lines show the estimated trajectory of eGFR. Group 1 shows the feature of rapid decliners, existed $14 \%$ of all. 
a straight line. Among group 1, 14\% of the participants had characteristics of early decliner. The predicted lowering speed of eGFR with group 1 at $\mathrm{T}$ years later was $3.5 \times \mathrm{T}-16$ $\left(\mathrm{mL} / \mathrm{min} / 1.73 \mathrm{~m}^{2} /\right.$ year $)$, which equaled the gradient of a tangent line to the original quadric curve, although that of group 2 was $-1.7\left(\mathrm{~mL} / \mathrm{min} / 1.73 \mathrm{~m}^{2} /\right.$ year $)$. The risk factors that contributed to position probability in group 1 are shown in table 3. Older age and a higher eGFR and ACR were significantly associated with early decliner status. Accordingly, the opposite trend were seen in group 3 (online supplementary table S11).

\section{DISCUSSION}

In a certain proportion of patients with diabetes, an eGFR decline precedes albuminuria. A new concept called DKD is advocated that also included the albuminuria-free patients. The prevalence of DKD in a large-scale database that integrated cohorts in Japan was indicated, and we revealed the risk factors of three subgroups of DKD. Additionally, we performed a longitudinal eGFR assessment to detect early decliners and the risk identification of early decliner using a trajectory analysis. Since the analysis has the advantage of proper classification by patients' eGFR variation consists of several different points, an exact assessment of eGFR decline rate of every group can be achieved. These findings must help the early detection of DKD and early decliners so that medical intervention can be provided at an early stage for high-risk patients.

In our multicenter study, approximately half of the patients with diabetes met the DKD criteria. Next, we divided patients with DKD into three groups to explore the risk factors by DKD category. Regarding atypical patients with low eGFR but normoalbuminuria, the prevalence was $23 \%$ in all patients with DKD. The status of normoalbuminuric patients with DKD was more strictly controlled with lifestyle-related disease than patients with albuminuria, and their eGFR decline rates were the slowest among all participant groups.

In a past study of the United States national observance of DKD,${ }^{16}$ the prevalence of DKD was $26 \%$. Although the prevalence of DKD, a lower eGFR $(<60 \mathrm{~mL} / \mathrm{min} / 1.73$ $\left.\mathrm{m}^{2}\right)(14 \%)$, and albuminuria (15\%) were lower than our findings, they were based on the doorstep screener of the general population; meanwhile, our data were provided by foundation hospitals including inpatients. Compared with past studies of DKD, ${ }^{51617}$ our participants showed better control of BMI and serum lipid status. On the other hand, glycemic control estimated by HbAlc and blood pressure (BP) were almost equivalent to those of previous studies. The patients were prescribed antidyslipidemic drugs $(52 \%)$ or antihypertensive drugs $(56 \%)$ at a frequency almost equal to those of patients with diabetes in a study from the USA. ${ }^{16}$ The suggestion that patients with diabetes in our facilities received therapeutic intervention for metabolic syndrome was almost equivalent to patients in the USA.
Although smaller percentage of patients with type 1 diabetes corresponded to DKD compared with type 2 diabetes, it cannot be generalized. A possible reason for the result is that patients with type 1 diabetes were followed up from an earlier stage of the disease compared with patients with type 2 diabetes.

For the decreased eGFR and normoalbuminuric category, clinical variables like HbA1c, BP, TG, and both HDL and LDL cholesterol levels were almost within the target range recommended by the Kidney Disease Improving Global Outcomes, American Diabetes Association, or the Kidney Disease Outcomes Quality Initiative guidelines ${ }^{14} 1819$ : HbAlc $\leq 7.0 \%(53 \mathrm{mmol} / \mathrm{mol}$ ) (aiming to prevent complications), BP $<140 / 90 \mathrm{~mm} \mathrm{Hg}, \mathrm{TG}<150$ $\mathrm{mg} / \mathrm{dL}, \mathrm{HDL} \geq 40 \mathrm{mg} / \mathrm{dL}$, and $\mathrm{LDL}<100 \mathrm{mg} / \mathrm{dL}$. Since those variables were not different from those of patients with no DKD, the patients with decreased eGFR were at lower risk of microangiopathy such as diabetic retinopathy. This is compatible with the fact that the prevalence of diabetes retinopathy in the decreased eGFR group was not different from that in the patients with no DKD. Their eGFR decline rates were slower than those of other patients with diabetes. It is deducible from the fact that they had a lower ratio of complications like diabetes retinopathy than the other DKD group, and they were prescribed antihypertensives at a significantly higher frequency than the patients with no DKD; $45 \%$ of the patients in that category were taking RAAS inhibitors, which might suppress the onset of albuminuria. ${ }^{20}$ They would benefit from the medication. Regarding UA, similar results were reported that the serum UA level predicted the decline of eGFR independently of other risk factors. ${ }^{21}$ Although the international criteria of metabolic syndrome ${ }^{22}$ does not include UA level, those patients with a higher UA level were much more likely to have metabolic syndrome. ${ }^{23}{ }^{24}$ It remains unknown why an elevated UA predicts an eGFR decline.

The group with albuminuria but a normal eGFR had a significantly higher ratio of a previous history of diabetic retinopathy and significantly higher HbAlc and SBP than patients with no DKD. This finding is compatible with disease phenotypes of microangiopathy as an early stage of classical diabetic nephropathy. Diabetic retinopathy is considered a risk factor of macroangiopathy according to the changes in vascular conductance and calcification. ${ }^{25}$ In particular, this group showed a higher prevalence of retinopathy than the other groups, suggesting a longer history of diabetes. ${ }^{5}$

These data implicated that a better past glycemic control, BP, and lipid condition might suppress albuminuria or microangiopathies like diabetic retinopathy. Strict long-term glycemic control reportedly suppressed the pathogenesis and progress of albuminuria in cases of type 1 diabetes. ${ }^{26}{ }^{27}$ Similarly, in patients with type 2 diabetes, strict glycemic control reduced the risk of microangiopathy and albuminuria. ${ }^{4}$ From recent studies, multidisciplinary therapy including glycemic, BP, and lipid control for type 2 diabetes significantly suppressed the pathogenesis and 
progress of albuminuria and decreased renal events like creatinine doubling ${ }^{28}$ or ESRD. ${ }^{29}$ Our results similarly indicated that good control of glycemic level, BP, and lipid condition might contribute to reducing renal damage and decreasing complications like microangiopathy. Our findings will be of great help in the early detection of patients with high-risk DKD and aid with early therapeutic intervention to avoid renal disorder.

Concerning the longitudinal analysis, we performed a trajectory analysis that could grasp yearly eGFR variations and classified the eGFR into three different patterns. An eGFR rapid lowering subgroup with eGFR normal baseline patients (14\%) was identified as early decliners. Their eGFR would decrease to $-29 \mathrm{~mL} / \mathrm{min} / 1.73 \mathrm{~m}^{2}$ within 4 years, and soon they may face the crisis of renal insufficiency. Their baseline factors such as older age, higher SBP, and ACR contributed to the eGFR decline. The same trend was seen when adding LDL cholesterol as a variable instead of total cholesterol (online supplementary table S9).

A higher ACR level reportedly predicts an eGFR decline and ESRD. ${ }^{3031}$

Regarding hypertension, despite several trials showing that strict BP control in the early stage of diabetes suppressed albuminuria onset or progression, there was no significant difference in eGFR decline within the follow-up term (4-8 years). ${ }^{32} 33$

However, an observational study of patients with hypertension but normal renal function showed that patients with poorly managed $\mathrm{BP}(\geq 160 / 95 \mathrm{~mm} \mathrm{Hg})$ experienced a rapid decline of eGFR compared with those with better controlled BP $(<140 / 90 \mathrm{~mm} \mathrm{Hg}){ }^{34}$ This finding is consistent with previous observations that poor BP control accelerates renal dysfunction.

Although a strong correlation existed between albuminuria and eGFR decline in our study, the eGFR reduction occurred despite baseline normoalbuminuria in a certain proportion of the patients with diabetes as seen in a previous report. ${ }^{35}$

These results will help us recognize early decliners by observing eGFR decline rate for approximately 3 years and risk factors such as a high SBP, high ACR, and older age to allow for early intervention in high-risk patients.

Although we excluded patients whose eGFR levels were considered too high, the definition of hyperfiltration remains obscure. Patients who underwent hyperfiltration reportedly had a high probability of a rapid eGFR decline. ${ }^{36}{ }^{37}$ In this study, we set our threshold of hyperfiltration as $120 \mathrm{~mL} / \mathrm{min} / 1.73 \mathrm{~m}^{237}$; however, regarding type 2 diabetes, it was difficult to completely exclude patients with hyperfiltration only by eGFR value because hyperfiltration would be hidden by age-related changes in eGFR in patients $>40$ years of age. ${ }^{36}$

This study has several limitations. First, due to its crosssectional observation design based on multicenter data, we could not avoid sampling bias. Because the baseline variables were not unified in this study, we could not elucidate the relevance of participant diabetes duration or complications other than renal damage, such as cardiovascular disorders, neuropathy, and malignancy. Second, we could not exclude various causes of renal damage except for diabetes since not all participants underwent a renal biopsy. It is possible that not all normoalbuminuric DKD can be actually attributed to DKD itself but can be a chronic kidney disease in patient with diabetes, which could be of multifactorial etiology like hypertension and atherosclerosis.

Moreover, the risk factors of DKD were calculated at only a moment in time; hence long-term follow-up in the future would reveal the characteristics of patients with DKD. In addition, we did not have enough data of prescription medicine including RAAS inhibitors to analyze the risk factors of DKD or early decliners although the data may affect the progression of renal damage.

However, our research included a large number and various types of patients with diabetes and analyzed the prevalence and risk factors of both DKD and early decliners using the same database.

In conclusion, here we revealed the epidemiology of DKD and identified past poor glycemic, SBP, and lipid control as risk factors of progression of renal damage and microangiopathy in patients with DKD. It is best to identify patients with DKD earlier and intervene in cases of hyperglycemia and other lifestyle problems in the early phase of diabetes.

\section{Author affiliations}

${ }^{1}$ Department of Biostatistics, The University of Tokyo, Bunkyo-ku, Tokyo, Japan ${ }^{2}$ Division of Nephrology and Endocrinology, The University of Tokyo, Bunkyo-ku, Tokyo, Japan

${ }^{3}$ Biostatistics Division, Central Coordinating Unit, Clinical Research Support Center, The University of Tokyo Hospital, Bunkyo-ku, Tokyo, Japan

${ }^{4}$ Department of Endocrinology, Metabolism and Diabetes, Faculty of Medicine, Kindai University, Osakasayama, Osaka, Japan

${ }^{5}$ Diabetes and Metabolism Information Center, Research Institute, National Center for Global Health and Medicine, Shinjuku-ku, Tokyo, Japan

${ }^{6}$ Diabetes Research Center, National Center for Global Health and Medicine,

Shinjuku-ku, Tokyo, Japan

${ }^{7}$ Department of Metabolism \& Endocrinology, Juntendo University Graduate School of Medicine, Bunkyo-ku, Tokyo, Japan

${ }^{8}$ Department of Diabetology \& Endocrinology, Kanazawa Medical University, Kahoku-gun, Ishikawa, Japan

${ }^{9}$ Department of Nephrology, Rheumatology, Endocrinology and Metabolism, Okayama University Graduate School of Medicine Dentistry and Pharmaceutical Sciences, Okayama, Japan

${ }^{10}$ Division of Nephrology, Kanazawa University Hospital, Ishikawa, Japan ${ }^{11}$ Department of Nephrology and Laboratory Medicine, Kanazawa University, Ishikawa, Japan

${ }^{12}$ Division of Clinical Nephrology and Rheumatology, Niigata University Graduate School of Medical and Dental Science Institute of Nephrology, Niigata, Japan ${ }^{13}$ Department of Nephrology and Hypertension, Kawasaki Medical School, Kurashiki, Okayama, Japan

Contributors YY contributed data interpretation, conducted statistical analysis, and wrote the first draft of the manuscript. KK and YM supervised programming for statistical analysis. YH, TT, MN, SN, HI, MO, KU, TW, TM, DK, KM, JW, MS, HW, YI, IN, and NK collected data, reviewed, and revised the manuscript.

Funding This work was supported by Japan Agency for Medical Research and Development under Grant Number JP19ek0210095h001.

Competing interests J-DREAMS is supported by Ministry of Health, Labour and Welfare, Japan Agency for Medical Research and Development, Japan Diabetes Society, Nippon Boehringer Ingelheim, Eli Lilly, Novo Nordisk Pharma, and Abbott Japan. KU reports lecture fees from Eli Lilly, Nippon Boehringer Ingelheim, 
Novo Nordisk, and Abbott Japan; grants and endowments from Eli Lilly, Nippon Boehringer Ingelheim, and Novo Nordisk.

Patient consent for publication Not required.

Provenance and peer review Not commissioned; externally peer reviewed.

Data availability statement The inspection and usage of the raw data in this study is restricted according to the ethical approval. All data relevant to the study are included in the article or uploaded as supplementary information.

Open access This is an open access article distributed in accordance with the Creative Commons Attribution Non Commercial (CC BY-NC 4.0) license, which permits others to distribute, remix, adapt, build upon this work non-commercially, and license their derivative works on different terms, provided the original work is properly cited, appropriate credit is given, any changes made indicated, and the use is non-commercial. See: http://creativecommons.org/licenses/by-nc/4.0/.

ORCID iDs

Yui Yoshida http://orcid.org/0000-0003-2764-073X

Shinsuke Noso http://orcid.org/0000-0002-0943-8450

Hiroshi Ikegami http://orcid.org/0000-0001-8808-4605

Tomoya Mita http://orcid.org/0000-0002-4401-2965

Jun Wada http://orcid.org/0000-0003-1468-5170

\section{REFERENCES}

1 Cho NH, Shaw JE, Karuranga S, et al. IDF Diabetes Atlas: global estimates of diabetes prevalence for 2017 and projections for 2045. Diabetes Res Clin Pract 2018;138:271-81.

2 Ministry of Health LaW, Japan. National health and nutrition examination survey 2016. Available: https://www.mhlw.go.jp/file/04Houdouhappyou-10904750-Kenkoukyoku-Gantaisakukenkouzous hinka/kekkagaiyou 7.pdf [Accessed 12 Feb 2019].

3 TJSfD T. The Japanese Society for dialysis therapy, illustration of present condition of Japanese maintenance dialysis 2016, 2016. Available: http://docs.jsdt.or.jp/overview/pdf2017/p017.pdf [Accessed 23 Nov 2018].

4 Adler Al, Stevens RJ, Manley SE, et al. Development and progression of nephropathy in type 2 diabetes: the United Kingdom Prospective Diabetes Study (UKPDS 64). Kidney Int 2003;63:225-32.

5 Penno G, Solini A, Orsi E, et al. Non-albuminuric renal impairment is a strong predictor of mortality in individuals with type 2 diabetes: the Renal Insufficiency And Cardiovascular Events (RIACE) Italian multicentre study. Diabetologia 2018;61:2277-89.

6 Yokoyama $\mathrm{H}$, Sone $\mathrm{H}$, Oishi $\mathrm{M}$, et al. Prevalence of albuminuria and renal insufficiency and associated clinical factors in type 2 diabetes: the Japan Diabetes Clinical Data Management study (JDDM15). Nephrol Dial Transplant 2009;24:1212-9.

7 de Boer IH, Rue TC, Hall YN, et al. Temporal trends in the prevalence of diabetic kidney disease in the United States. JAMA 2011;305:2532-9

8 Perkins BA, Ficociello LH, Ostrander BE, et al. Microalbuminuria and the risk for early progressive renal function decline in type 1 diabetes. J Am Soc Nephrol 2007;18:1353-61.

9 Skupien J, Warram JH, Smiles AM, et al. The early decline in renal function in patients with type 1 diabetes and proteinuria predicts the risk of end-stage renal disease. Kidney Int 2012;82:589-97.

10 Zoppini G, Targher G, Chonchol M, et al. Predictors of estimated GFR decline in patients with type 2 diabetes and preserved kidney function. Clin J Am Soc Nephrol 2012;7:401-8.

11 Jiang G, Luk AOY, Tam CHT, et al. Progression of diabetic kidney disease and trajectory of kidney function decline in Chinese patients with type 2 diabetes. Kidney Int 2019;95:178-87.

12 Society TJD. Diabetes treatment guideline 2018-2019: Bunkodo, 2018.

13 JSo N. Clinical practice guidebook for diagnosis and treatment of chronic kidney disease: Tokyo Igakusha, 2012.

14 Inker LA, Astor BC, Fox CH, et al. KDOQI US commentary on the $2012 \mathrm{KDIGO}$ clinical practice guideline for the evaluation and management of CKD. Am J Kidney Dis 2014;63:713-35.

15 Burckhardt P, Nagin DS, Padman R. Multi-Trajectory models of chronic kidney disease progression. AMIA Annu Symp Proc 2016;2016:1737-46.
16 Afkarian M, Zelnick LR, Hall YN, et al. Clinical manifestations of kidney disease among US adults with diabetes, 1988-2014. JAMA 2016;316:602-10.

17 New JP, Middleton RJ, Klebe B, et al. Assessing the prevalence, monitoring and management of chronic kidney disease in patients with diabetes compared with those without diabetes in general practice. Diabet Med 2007;24:364-9.

18 American Diabetes Association. Standards of Medical Care in Diabetes-2018 Abridged for Primary Care Providers. Clin Diabetes 2018;36:14-37.

19 KDOQI. KDOQI clinical practice guidelines and clinical practice recommendations for diabetes and chronic kidney disease. Am J Kidney Dis 2007:49:S12-154.

20 Casas JP, Chua W, Loukogeorgakis S, et al. Effect of inhibitors of the renin-angiotensin system and other antihypertensive drugs on renal outcomes: systematic review and meta-analysis. Lancet 2005;366:2026-33.

21 De Cosmo S, Viazzi F, Pacilli A, et al. Serum uric acid and risk of CKD in type 2 diabetes. Clin J Am Soc Nephrol 2015;10:1921-9.

22 Alberti KGMM, Eckel RH, Grundy SM, et al. Harmonizing the metabolic syndrome: a joint interim statement of the International Diabetes Federation Task Force on Epidemiology and Prevention; National Heart, Lung, and Blood Institute; American Heart Association; World Heart Federation; International Atherosclerosis Society; and International Association for the Study of Obesity. Circulation 2009:120:1640-5.

23 Onat $A$, Uyarel $H$, Hergenç $G$, et al. Serum uric acid is a determinant of metabolic syndrome in a population-based study. Am J Hypertens 2006; 19:1055-62.

24 Lohsoonthorn V, Dhanamun B, Williams MA. Prevalence of hyperuricemia and its relationship with metabolic syndrome in Thai adults receiving annual health exams. Arch Med Res 2006;37:883-9.

25 Gerstein HC, Ambrosius WT, Danis R, et al. Diabetic retinopathy, its progression, and incident cardiovascular events in the ACCORD trial. Diabetes Care 2013;36:1266-71.

26 The effect of intensive diabetes therapy on the development and progression of neuropathy. The Diabetes Control and Complications Trial Research Group. Ann Intern Med 1995;122:561-8.

27 Nathan DM, Cleary PA, Backlund J-YC, et al. Intensive diabetes treatment and cardiovascular disease in patients with type 1 diabetes. N Engl J Med 2005;353:2643-53.

28 Ueki K, Sasako T, Okazaki Y, et al. Effect of an intensified multifactorial intervention on cardiovascular outcomes and mortality in type 2 diabetes (J-DOIT3): an open-label, randomised controlled trial. Lancet Diabetes Endocrinol 2017:5:951-64.

29 Gaede $\mathrm{P}$, Lund-Andersen $\mathrm{H}$, Parving $\mathrm{H}-\mathrm{H}$, et al. Effect of a multifactorial intervention on mortality in type 2 diabetes. $N$ Engl J Med 2008:358:580-91.

30 Iseki K, Ikemiya Y, Iseki C, et al. Proteinuria and the risk of developing end-stage renal disease. Kidney Int 2003;63:1468-74.

31 Lea J, Greene T, Hebert L, et al. The relationship between magnitude of proteinuria reduction and risk of end-stage renal disease: results of the African American study of kidney disease and hypertension. Arch Intern Med 2005;165:947-53.

32 Gansevoort RT, Matsushita K, van der Velde M, et al. Lower estimated GFR and higher albuminuria are associated with adverse kidney outcomes. A collaborative meta-analysis of general and highrisk population cohorts. Kidney Int 2011;80:93-104.

33 Tight blood pressure control and risk of macrovascular and microvascular complications in type 2 diabetes: UKPDS 38. UK prospective diabetes Study Group. BMJ 1998;317:703-13.

34 Vupputuri S, Batuman V, Muntner P, et al. Effect of blood pressure on early decline in kidney function among hypertensive men. Hypertension 2003;42:1144-9.

35 Porrini E, Ruggenenti P, Mogensen CE, et al. Non-proteinuric pathways in loss of renal function in patients with type 2 diabetes. Lancet Diabetes Endocrinol 2015;3:382-91.

36 Silveiro SP, Friedman R, de Azevedo MJ, et al. Five-Year prospective study of glomerular filtration rate and albumin excretion rate in normofiltering and hyperfiltering normoalbuminuric NIDDM patients. Diabetes Care 1996;19:171-4.

37 Moriya T, Tanaka S, Sone H, et al. Patients with type 2 diabetes having higher glomerular filtration rate showed rapid renal function decline followed by impaired glomerular filtration rate: Japan Diabetes Complications Study. J Diabetes Complications $2017 ; 31: 473-8$ 


\section{Correction: Conditions, pathogenesis, and progression of diabetic kidney disease and early decliner in Japan}

Yoshida Y, Kashiwabara K, Hirakawa Y, et al. Conditions, pathogenesis, and progression of diabetic kidney disease and early decliner in Japan. BMJ Open Diab Res Care 2020;8:e000902. doi: 10.1136/bmjdrc-2019-000902

This article was previously published with an error.

1. The formula for predicting the rate of lowering speed of eGFR at $\mathrm{T}$ years later in trajectory analysis was incorrect (Results, P6, line 3).

In the actual data, $T$ was calculated as a value of 1 (baseline) to 5 instead of 0 to 4 . Correct: $3.5 \times(\mathrm{T}+1)-16$

Incorrect: $3.5 \times \mathrm{T}-16$

2. The eGFR value 4 years after from baseline of the early decliner predicted by trajectory analysis was incorrect (Discussion, P7, line 14).

The formula predicted by trajectory analysis for predicted eGFR after $\mathrm{T}$ years is below (not shown in manuscript):

$\mathrm{F}(\mathrm{T}+1)=13.560539-15.968622 *(\mathrm{~T}+1)+1.767836 *(\mathrm{~T}+1)^{2}$

The eGFR value after 4 years is derived to be $-22 \mathrm{~mL} / \mathrm{min} / 1.73 \mathrm{~m}^{2}$, which is in agreement with the results shown in the figure 2 (P5).

Correct: $-22 \mathrm{~mL} / \mathrm{min} / 1.73 \mathrm{~m}^{2}$

Incorrect: $-29 \mathrm{~mL} / \mathrm{min} / 1.73 \mathrm{~m}^{2}$

(2)

\section{OPEN ACCESS}

Open access This is an open access article distributed in accordance with the Creative Commons Attribution Non Commercial (CC BY-NC 4.0) license, which permits others to distribute, remix, adapt, build upon this work noncommercially, and license their derivative works on different terms, provided the original work is properly cited, appropriate credit is given, any changes made indicated, and the use is non-commercial. See: http://creativecommons. org/licenses/by-nc/4.0/.

C Author(s) (or their employer(s)) 2020. Re-use permitted under CC BY-NC. No commercial re-use. See rights and permissions. Published by BMJ.

BMJ Open Diab Res Care 2020;8:e000902corr1. doi:10.1136/bmjdrc-2019-000902corr1

A) Check for updates 